\title{
ARTICLE Density of small dendritic spines and microtubule-associated- protein-2 immunoreactivity in the primary auditory cortex of subjects with schizophrenia
}

Brandon C. McKinney $\mathbb{I D}^{1,2}$, Matthew L. MacDonald ${ }^{1,2}$, Jason T. Newman ${ }^{2}$, Micah A. Shelton ${ }^{2}$, Rebecca A. DeGiosio ${ }^{2,3}$, Ryan M. Kelly ${ }^{4}$, Kenneth N. Fish ${ }^{1,2}$, Allan R. Sampson ${ }^{4}$, David A. Lewis ${ }^{1,2,3}$ and Robert A. Sweet $\mathbb{D}^{1,2,5,6}$

Previously, we demonstrated that dendritic spine density (DSD) in deep layer 3 of the primary auditory cortex (A1) is lower, due to having fewer small spines, in subjects with schizophrenia (SZ) than non-psychiatric control (NPC) subjects. We also previously demonstrated that microtubule-associated-protein-2 immunoreactivity (MAP2-IR) in A1 deep layer 3 is lower, and positively correlated with DSD, in SZ subjects. Here, we first sought to confirm these findings in an independent cohort of 25 SZ-NPC subject pairs (cohort 1). We used immunohistochemistry and confocal microscopy to measure DSD and MAP2-IR in A1 deep layer 3. Consistent with previous studies, both DSD and MAP2-IR were lower in SZ subjects. We then tested the hypothesis that MAP2-IR mediates the effect of SZ on DSD in a cohort of 45 SZ-NPC subject pairs (combined cohort) that included all subjects from cohort 1 and two previously studied cohorts. Based on the distribution of MAP2-IR values in NPC subjects, we categorized each SZ subject as having either low MAP2-IR (SZ MAP2-IR(low)) or normal MAP2-IR (SZ MAP2-IR(normal)). Among SZ MAP-IR(low) subjects, mean DSD was significantly lower than in NPC subjects. However, mean DSD did not differ between SZ MAP2-IR(normal) and NPC subjects. Moreover, MAP2-IR statistically mediated small spine differences, with lower MAP2-IR values associated with fewer small spines. Our findings confirm that low density of small spines and low MAP2-IR are robust SZ phenotypes and suggest that MAP2-IR mediates the effect of SZ on DSD.

Neuropsychopharmacology (2019) 44:1-7; https://doi.org/10.1038/s41386-019-0350-7

\section{INTRODUCTION}

A dendritic spine (or spine) is a small, actin-rich structure that protrudes from neuronal dendrites, and comprises the postsynaptic site of most excitatory glutamatergic synapses [1]. Spines are intimately linked to neuron function. Dynamic changes in spine number are essential for normal cognition and sensory processing [2-4], and many disorders characterized by impaired cognition and abnormal sensory processing are associated with alterations in dendritic spine density (DSD) [5].

The dendrites of pyramidal neurons in the human cerebral cortex are covered with spines, and lower DSD on cortical layer 3 pyramidal neurons is consistently observed in postmortem studies of schizophrenia (SZ) subjects [6, 7], thus suggesting disruption of the circuits in which these neurons participate. Particular symptoms of SZ are thought to be associated with disruptions of certain cortical circuits [8]. For example, cortical circuit abnormalities in primary auditory cortex (A1), a brain region critical for auditory processing, are associated with auditory verbal hallucinations and impaired auditory sensory processing. Impaired auditory processing further contributes to phonologic dyslexia and difficulty recognizing and expressing spoken emotional tone (prosody) in subjects with SZ [9]. In this, and previous studies, we have focused on better understanding $A 1$ abnormalities in SZ subjects. We previously demonstrated, in three independent cohorts, that DSD is lower in A1 deep layer 3 of subjects with SZ $[10,11]$, and showed that this is due to fewer small spines [12].

Microtubule (MT)-associated-protein-2 (MAP2) is a neuronspecific cytoskeletal protein expressed in the soma and dendrites of mature neurons [13]. MAP2 was originally characterized by its ability to bind and stabilize MTs in dendrites [14], but is now known to regulate additional MT functions, including the effects of MTs on spine morphology [15]. Dynamic MTs enter spines in response to synaptic activity [16], and during spine development, leading to a transition from immature filopodia to a mature spine [17]. In mature spines, MT entry protects against spine size reductions induced by long-term depression [15]. The link between MAP2 and spine structural plasticity is further supported by findings that inhibition of MT polymerization leads to impaired long-term potentiation, regression of mature-appearing spines to immature spine shapes, and loss of spines [16-18].

Here, we sought to first confirm our previous DSD and MAP2-IR findings in a larger, independent cohort of 25 subjects with SZ and 25 matched non-psychiatric control (NPC) subjects. We then tested our hypothesis that MAP2-IR mediates the effect of SZ on

\footnotetext{
${ }^{1}$ Department of Psychiatry, University of Pittsburgh, Pittsburgh, PA, USA; ${ }^{2}$ Translational Neuroscience Program, University of Pittsburgh, Pittsburgh, PA, USA; ${ }^{3}$ Department of

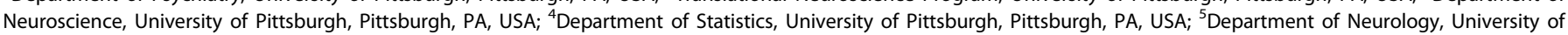
Pittsburgh, Pittsburgh, PA, USA and ${ }^{6}$ Mental Illness Research, Education, and Clinical Center, VA Pittsburgh Healthcare System, Pittsburgh, PA, USA

Correspondence: Robert A. Sweet (sweetra@upmc.edu)

Web: http://www.wpic.pitt.edu/research/sweetlab/
}

Received: 25 October 2018 Revised: 15 February 2019 Accepted: 16 February 2019

Published online: 22 February 2019 
Table 1. Cohort characteristics

\begin{tabular}{|c|c|c|c|c|c|c|c|c|}
\hline \multirow{2}{*}{$\begin{array}{l}\text { Cohort } \\
\text { Diagnostic group }\end{array}$} & \multicolumn{2}{|l|}{1} & \multicolumn{2}{|l|}{2} & \multicolumn{2}{|l|}{3} & \multicolumn{2}{|l|}{ Combined } \\
\hline & NPC & SZ & NPC & SZ & NPC & SZ & NPC & SZ \\
\hline Number & 25 & 25 & 12 & 12 & 8 & 8 & 45 & 45 \\
\hline Sex & $20 \mathrm{M}, 5 \mathrm{~F}$ & $20 \mathrm{M}, 5 \mathrm{~F}$ & $9 \mathrm{M}, 3 \mathrm{~F}$ & $9 \mathrm{M}, 3 \mathrm{~F}$ & $4 \mathrm{M}, 4 \mathrm{~F}$ & $4 \mathrm{M}, 4 \mathrm{~F}$ & $33 \mathrm{M}, 12 \mathrm{~F}$ & $33 \mathrm{M}, 12 \mathrm{~F}$ \\
\hline Race & $21 \mathrm{~W}, 4 \mathrm{~B}$ & $22 W, 3 B$ & $8 \mathrm{~W}, 3 \mathrm{~B}, 10$ & $8 W, 4 B$ & $8 W$ & $8 W$ & $36 \mathrm{~W}, 8 \mathrm{~B}, 10$ & $35 \mathrm{~W}, 10 \mathrm{~B}$ \\
\hline Age (years) & $50.68 \pm 2.83$ & $48.48 \pm 2.97$ & $45.2 \pm 3.72$ & $47.25 \pm 3.86$ & $46.38 \pm 4.94$ & $46.5 \pm 4.38$ & $48.44 \pm 2.04$ & $47.80 \pm 2.06$ \\
\hline PMI (hours) & $18.48 \pm 0.95$ & $19.43 \pm 1.33$ & $18.12 \pm 1.87$ & $17.92 \pm 2.53$ & $13.75 \pm 2.30$ & $15.63 \pm 2.39$ & $17.54 \pm 0.85$ & $18.35 \pm 1.08$ \\
\hline $\mathrm{pH}$ & $6.63 \pm 0.05$ & $6.52 \pm 0.06$ & $6.87 \pm 0.05$ & $6.51 \pm 0.09$ & $6.51 \pm 0.06$ & $6.47 \pm 0.12$ & $6.67 \pm 0.04$ & $6.51 \pm 0.04$ \\
\hline $\begin{array}{l}\text { Storage time } \\
\text { (months) }\end{array}$ & $140.00 \pm 8.04$ & $136.70 \pm 9.98$ & $145.48 \pm 7.85$ & $154.99 \pm 8.59$ & $97.08 \pm 7.93$ & $92.76 \pm 4.96$ & $136.40 \pm 5.83$ & $131.2 \pm 6.58$ \\
\hline
\end{tabular}

Data for continuous variables are presented as "group average plus or minus standard error of the mean"

NPC non-psychiatric control, SZ schizophrenia, M male, $F$ female, PMI postmortem interval, $W$ white, $B$ black, $O$ other (Asian Indian)

DSD in a combined cohort with subjects from the cohort studied here and two previously studied cohorts. Finally, we performed analyses to verify that the DSD and MAP2-IR phenotypes are not associated with potential confounding variables.

\section{METHODS}

Human subjects

Tissue was obtained from postmortem brains recovered during autopsies conducted at the Allegheny County Medical Examiner's Office, Pittsburgh, PA, following informed consent from the next of kin. All procedures were approved by the University of Pittsburgh Committee for the Oversight of Research and Clinical Trials Involving the Dead and the Institutional Review Board for Biomedical Research as previously described [10, 19]. An independent committee of experienced clinicians made consensus Diagnostic and Statistical Manual of Mental Disorders, Fourth Edition diagnoses, or absence thereof, for each subject using medical records, structured interviews with surviving relatives, and, when available, toxicology reports [20].

\section{Cohort membership}

DSD and MT-associated-protein-2 immunoreactivity (MAP-IR) were measured in a new cohort (cohort 1) comprising subjects diagnosed with SZ $(n=17)$ or schizoaffective disorder $(n=8)$, and 25 NPC subjects. Subjects diagnosed with SZ and schizoaffective disorder were grouped together (henceforth referred to as SZ) for analysis as our previous studies have found that neither DSD nor MAP2-IR differ between the diagnoses $[10,11]$. SZ and NPC subjects were matched for sex, and as closely as possible for both age and post-mortem interval (PMI). A potential mediating role for MAP2-IR in the SZ DSD phenotype and the effects of potential confounding variables on DSD and MAP2-IR were assessed in a combined cohort comprising cohort 1 and two previously studied cohorts (cohorts 2 and 3). SZ and NPC groups did not differ significantly with respect to PMI, $\mathrm{pH}$, or storage time in either cohort 1 or the combined cohort (Table 1 and Supplemental Table 1).

Immunohistochemistry

To visualize spines, two markers were used in combination: a polyclonal antibody directed against spinophilin (EMD Millipore Corporation, Temecula, CA, USA AB5669), and the filamentous actin (F-actin) binding mushroom toxin phalloidin conjugated to Alexa Fluor 568 (3 U/ml; Invitrogen, Carlsbad, CA, USA, A12380). Spinophilin is a phosphatase-1-binding protein localized to spines $[21,22]$, and F-actin is a cytoskeleton protein enriched in spines [23]. MAP2 was labeled with the mouse monoclonal antibody SMI52 (1:500; BioLegend Inc., San Diego, CA, USA, 801801). SMI-52 recognizes all four major MAP2 isoforms (ref. [24], see also
Supplemental Fig. 1) (see Supplemental Methods for additional details).

Image collection

Sections from subject pairs were imaged together, in a randomized block design. Sections were aligned to the contours drawn on adjacent Nissl-stained sections. Sites within deep layer 3 were then selected using a randomly rotated sampling grid generated within Stereo Investigator, yielding a total of 20 sites sampled per subject. At each site, images were collected with a 1.40 numerical aperture $\times 60$ oil supercorrected objective (see Supplemental Methods for equipment details).

At each sampling site, sequential image planes separated by $0.25 \mu \mathrm{m}$ beginning $12.5 \mu \mathrm{m}$ below the tissue surface closest to the coverglass and ending at the tissue surface/coverslip interface were collected as $3-D$ data sets. Using this approach, $512 \times 512 \times$ 50 -voxel image stacks were generated. For $488 \mathrm{~nm}$ (spinophilin) and $568 \mathrm{~nm}$ (phalloidin) excitation wavelengths, exposure times were set to optimize the spread of the intensity histogram at each site, and constant exposure was used at $647 \mathrm{~nm}$ (MAP2).

Image processing

Slidebook software version 6.010 (Intelligent Imaging Innovations Inc., Denver, CO, USA) with keystrokes automated by the Automation Anywhere software (Automation Anywhere Inc., San Jose, CA, USA) was used to process all images. Image stacks were deconvolved using AutoQuant's blind deconvolution algorithm (MediaCybernetics, Rockville, MD, USA). After deconvolution, a Gaussian channel was made for the spinophilin and phalloidin deconvolved channels by calculating a difference of Gaussians using sigma values of 0.7 and 2. The Gaussian channel, which demarcates the edges of immunofluorescence puncta, was used for data segmentation only [25]. Segmentation of the spinophilin and phalloidin Gaussian channels was performed as previously described [26]. Briefly, the Ridler-Calvard iterative thresholding algorithm [27] was used to obtain an initial value for iterative segmentation for each channel within each image stack. Multiple iterations with subsequent threshold settings increasing by 25 gray levels were performed in MATLAB (R2015b). After each iteration, the object masks were size gated within a range of 0.1 and $0.8 \mu^{3}$ (spinophilin channel) and 0.04 and $1.5 \mu^{3}$ (phalloidin channel). The final object masks for each channel were then used to collect information on the deconvolved channels and determine DSD. The definition of a spine was operationalized as a spinophilin-immunoreactive mask object that overlaps ( $\geq 1$ voxel) a phalloidin mask object. For the MAP2 channel, a single-plane $2 \mu \mathrm{m}$ beneath the tissue surface at the same sites where images stacks were captured was masked using the Ridler-Calvard iterative thresholding algorithm [27], and MAP2 intensity within 
the masked objects extracted (see Supplemental Methods for additional details).

Statistical analyses

Confirming the effect of SZ on DSD and MAP2 immunoreactivity. To confirm that the diagnostic effect on DSD and MAP2-IR in cohort 1 was similar to the previously observed effects in cohorts 2 and 3, simple linear models were used as previously described [12]. For each subject, both overall DSD and DSD within a size category were assumed to be normally distributed, and MAP2-IR for each subject was log-transformed to more normally distribute the data. These analyses were repeated for the combined cohort.

Establishing the mediating role of MAP2 immunoreactivity in the effect of SZ on DSD. Under the Baron and Kenny approach for establishing mediation [28], four criteria must be met in a given spine size category to conclude that MAP2-IR plays a mediating role in that category:

1. Without controlling for MAP2-IR, diagnosis predicts DSD.

2. MAP2-IR levels differ between diagnostic groups.

3. Controlling for MAP2-IR, there is a significant positive relationship between DSD and MAP2-IR.

4. The estimated effect sizes of diagnosis on DSD are larger in absolute value in the model without MAP2-IR than the model with MAP2-IR.
A linear mixed model containing Pair, Cohort, Diagnosis, Spine Size Category, Cohort*Diagnosis, Cohort*Spine Size Category, Diagnosis*Spine Size Category, and Cohort*Diagnosis*Spine Size Category fixed effects and Subject normal random effect was used to estimate and test the effect of Diagnosis on DSD without controlling for MAP2-IR. Insignificant interaction terms were removed from the model. Bonferroni-corrected $p$ values were obtained for the tests of no diagnostic effect in each of the 10 spine size categories. A similar approach to the DSD analysis was performed for MAP2-IR, without including Spine Size Category.

A mixed-effects model for DSD within size category was fit containing Pair, Cohort, Diagnosis, Spine Size Category, MAP2-IR, Cohort*Spine Size Category, Diagnosis*Spine Size Category, and Spine Size Category*MAP2-IR*Cohort fixed effects, and Subject random effect. Bonferroni-corrected $p$ values are given for the mediated spine size categories, which were found significant in the DSD analyses by category. There were indications of a Spine Size Category*MAP2-IR*Cohort interaction of a quantitative nature, with no apparent qualitative differences by Cohort. Thus, the analyses that led to Table 2 and the mediation determination allowed for no interactions by Cohort. To obtain the most accurate estimates of the indirect mediation effects, we chose to allow the effect of MAP2-IR to vary among cohorts by adding MAP2$I R^{*}$ Cohort and MAP2-IR*Cohort*Spine Size Category fixed effects into the model. In effect this approach to determining the indirect

\begin{tabular}{|c|c|c|c|c|c|c|c|}
\hline \multirow{3}{*}{ Spine size bins $\left(\mu \mathrm{m}^{3}\right)$} & \multirow{3}{*}{ Variables } & \multicolumn{2}{|l|}{1} & \multicolumn{2}{|l|}{2} & \multicolumn{2}{|l|}{3} \\
\hline & & \multicolumn{2}{|c|}{ Diagnosis only* } & \multicolumn{2}{|c|}{ MAP2-IR only* } & \multicolumn{2}{|c|}{ Diagnosis and MAP2-IR* } \\
\hline & & Coefficient & $p$ value & Coefficient & $p$ value & Coefficient & $p$ value \\
\hline \multirow[t]{2}{*}{$0.15-0.3$} & Diagnosis (SZ-NPC) & -0.00172 & $<0.001^{\mathrm{a}}$ & & & -0.000943 & $0.0024^{b}$ \\
\hline & MAP2-IR & & & 0.001114 & $<0.001^{\mathrm{a}}$ & 0.000801 & $<0.0003^{b}$ \\
\hline \multirow[t]{2}{*}{$0.3-0.45$} & Diagnosis (SZ-NPC) & -0.001044 & $<0.001^{\mathrm{a}}$ & & & -0.000596 & $0.0981^{\mathrm{b}}$ \\
\hline & MAP2-IR & & & 0.000814 & $<0.001^{a}$ & 0.000462 & $0.0231^{b}$ \\
\hline $0.45-0.60$ & Diagnosis (SZ-NPC) & -0.000558 & $0.177^{\mathrm{a}}$ & & & -0.000258 & 0.3511 \\
\hline \multirow[t]{2}{*}{$0.75-0.90$} & Diagnosis (SZ-NPC) & -0.000176 & $1^{\mathrm{a}}$ & & & 0.000027 & 0.9277 \\
\hline & MAP2-IR & & & 0.000248 & $0.9151^{\mathrm{a}}$ & 0.000209 & 0.219 \\
\hline \multirow[t]{2}{*}{$0.90-1.05$} & Diagnosis (SZ-NPC) & -0.000127 & $1^{\mathrm{a}}$ & & & 0.000052 & 0.8499 \\
\hline & MAP2-IR & & & 0.000201 & $1^{\mathrm{a}}$ & 0.000185 & 0.2777 \\
\hline \multirow[t]{2}{*}{$1.05-1.20$} & Diagnosis (SZ-NPC) & -0.000076 & $1^{\mathrm{a}}$ & & & 0.000115 & 0.677 \\
\hline & MAP2-IR & & & 0.000175 & $1^{\mathrm{a}}$ & 0.000197 & 0.2481 \\
\hline \multirow[t]{2}{*}{$1.20-1.35$} & Diagnosis (SZ-NPC) & -0.000054 & $1^{\mathrm{a}}$ & & & 0.000119 & 0.6677 \\
\hline & MAP2-IR & & & 0.000148 & $1^{\mathrm{a}}$ & 0.000177 & 0.297 \\
\hline \multirow[t]{2}{*}{$>1.35$} & Diagnosis (SZ-NPC) & -0.000026 & $1^{\mathrm{a}}$ & & & 0.00019 & 0.4932 \\
\hline & MAP2-IR & & & 0.000218 & $1^{\mathrm{a}}$ & 0.000222 & 0.1932 \\
\hline
\end{tabular}


mediation effects is like assuming a common effect of MAP2-IR across Cohorts and using a single "meta-analytic" estimate of the effect of MAP2-IR on DSD. Estimates of the indirect effect were obtained by subtracting the estimates of the diagnostic effect in this model from the estimates in the first model. Then, the significance of the indirect mediation effects are tested using bootstrapping on pairs within the cohort in 1000 samples. The mediation effect is significant if its $95 \%$ confidence intervals (Cls) do not include zero [29, 30]. Denominator degrees of freedom for the models were calculated using the Kenward-Roger method. The analyses were implemented in SAS 9.4 using PROC MIXED.

Assessing the effects of potential confounding variables on DSD and $M A P 2-I R$. To assess the effects of the potential categorical confounding variables sex, schizoaffective disorder, suicide, cannabis use disorder history, and the presence ("Yes" versus "No") at the time of death of each of several medication/substances (tobacco, antipsychotics, anticonvulsants, antidepressants, benzodiazepines, and other medications) on DSD and MAP2-IR, the percent difference in DSD or MAP2-IR in every spine size category within each pair was calculated (NPC-SZ)/NPC*100\%). These values were compared between the "Yes" group and the "No" group using a two-sample $t$ test with equal variances (Supplemental Table 2). The presence of each medication/substance at the time of death was determined most often based on review of medical records and interviews with next of kin. On the few occasions that results of postmortem toxicology studies were available, they were used to aid in this determination.

In the case of the potential continuous confounding variables, age of onset and duration of illness, a linear regression was performed with percent difference in DSD or MAP2-IR, and the potential confounding variable as the dependent and independent variables, respectively (Supplemental Table 2 ).

\section{RESULTS}

\section{Cohort 1}

Density of small dendritic spines and MAP2-IR are lower in deep layer 3 of the primary auditory cortex of SZ subjects. Mean DSD in deep layer 3 of $A 1$ in SZ subjects was significantly lower by $14 \%$ (NPC = $0.044 \pm 0.0016$ spines $/ \mu \mathrm{m}^{3} ; \mathrm{SZ}=0.038 \pm 0.0016$ spines $\left./ \mu \mathrm{m}^{3}\right)(F(1$, $48)=4.95, p<0.05$, Fig. 1b). After distributing spines into categories based on spine volume $\left(0.15 \mu \mathrm{m}^{3}\right.$ increments with the final size category including all spines with volume $>1.35 \mu^{3}$ ) (Fig. 1c), DSD was lower in only in the three categories representing the small spines $\left(<0.45 \mu \mathrm{m}^{3}\right)$, but not in the categories representing medium and large spines $\left(\geq 0.45 \mu \mathrm{m}^{3}\right)$ (Fig. 1d), thus replicating our previous findings in cohorts 2 and 3 (Fig. 1e) [12].

Mean MAP2-IR was $48 \%$ lower in deep layer 3 of A1 in SZ subjects $(S Z=1147 \pm 218$ grayscale levels; $N P C=2209 \pm 279$ grayscale levels) $(F(1,48)=9.97, p<0.001$, Fig. 2$)$.

\section{Combined cohort}

DSD and MAP2-IR phenotypes persist in combined cohort and do not associate with potential confounding variables. Because the same methods for quantifying DSD and MAP2-IR were used in all three cohorts [11, 12], we combined the data from each for further analysis. In the combined cohort, mean DSD was lower by $16 \%$ $\left(\mathrm{NPC}=0.039 \pm 0.0014\right.$ spines $/ \mu \mathrm{m}^{3} ; \mathrm{SZ}=0.033 \pm 0.0015$ spines $/$ $\left.\mu^{3}\right)(F(1,88)=4.40, p<0.05)$, and mean MAP2-IR was lower by $53 \%$ in deep layer 3 of A1 from SZ subjects (NPC $=1969 \pm 255$ grayscale levels; $S Z=923 \pm 150$ grayscale levels $)(F(1,88)=21.19$, $p<0.001)$. Importantly, we found no association of potential clinical confounding variables with either DSD or MAP2-IR, despite the greater power to detect smaller effects in the combined cohort (Supplemental Table 2).
A

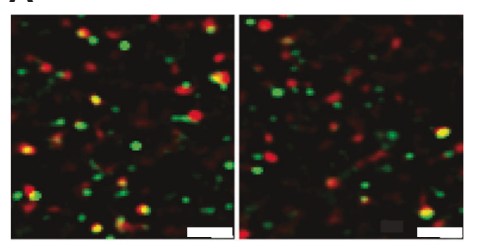

B

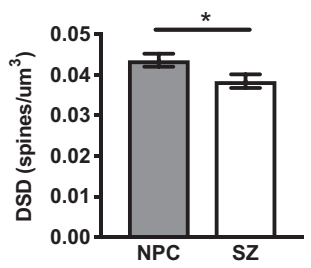

C

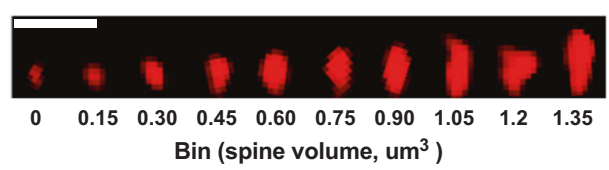

D
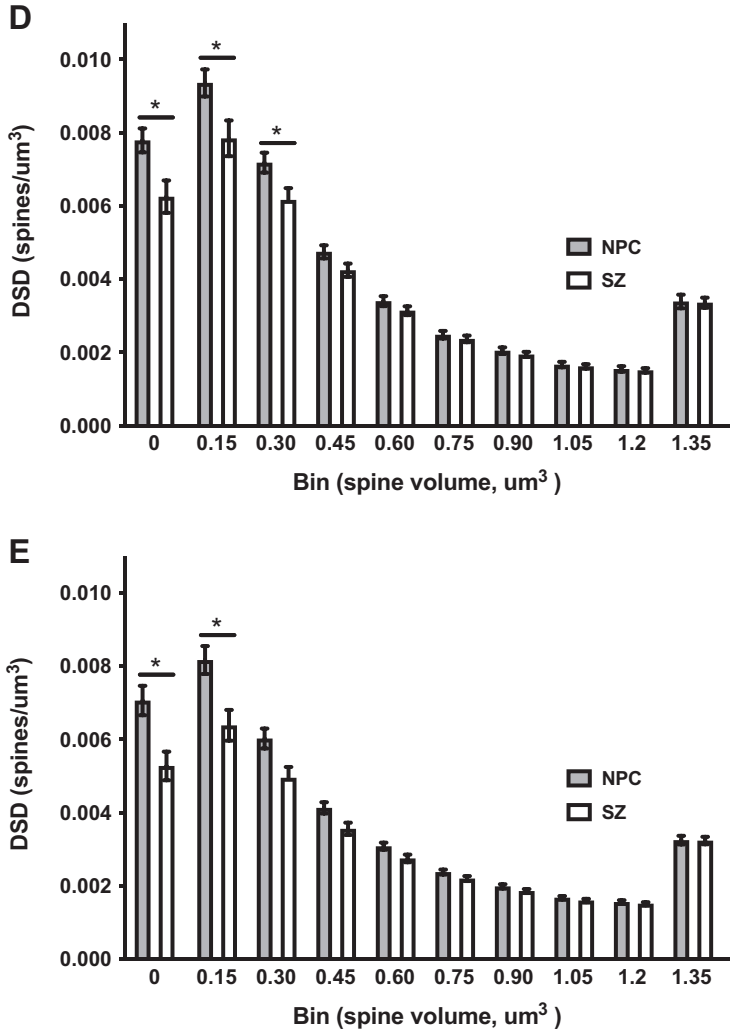

Fig. 1 Density of small dendritic spines is lower in primary auditory cortex deep layer 3 of schizophrenia subjects. a A spine was defined as the colocalization of spinophilin-immunoreactive puncta (green) and phalloidin-labeled puncta (red). Representative micrographs from a non-psychiatric control (NPC) subject (left) and a schizophrenia (SZ) subject (right). Scale bar $=2 \mu \mathrm{m}$. b Mean dendritic spine density (DSD) in deep layer 3 of the primary auditory cortex was lower in SZ subjects. c Representative micrographs of spines in each spine size category to which spines were distributed based on volume $\left(0.15 \mu \mathrm{m}^{3}\right.$ increments with the final size category including all spines with volume $>1.35 \mu \mathrm{m}^{3}$ ). Scale bar $=2 \mu \mathrm{m}$. d DSD in the small size categories (volume $<0.45 \mu \mathrm{m}^{3}$ ) was lower in SZ subjects (e), thus replicating our previous findings in cohorts 2 and 3 (adapted from ref. [12]). Error bars represent \pm standard error of the mean, and * indicates Bonferroni-corrected $p<0.05$

Low mean MAP2-IR in the SZ group is due to a subgroup of subjects with low values. To further explore the relationship between DSD and MAP2-IR, we divided SZ subjects into a low MAP2-IR group (SZ MAP2-IR(low)) and normal MAP2-IR group (SZ MAP2-IR(normal)) determined by the 25th percentile MAP2-IR values in NPC subjects $(\operatorname{In}(M A P 2-I R)=6.52) \quad$ (Fig. 3a, b). Sixty percent (27/45) of SZ subjects were classified as MAP2-IR(low). Significantly lower DSD 


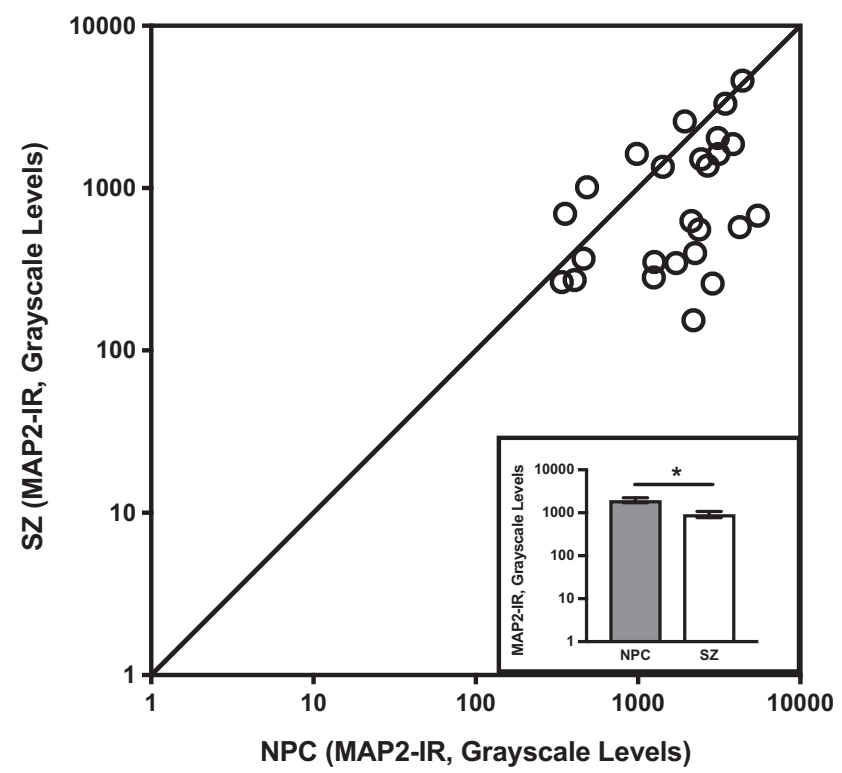

Fig. 2 Microtubule-associated-protein-2 immunoreactivity (MAP2IR) is lower in deep layer 3 of the primary auditory cortex of schizophrenia (SZ) subjects. (Main) Relative MAP2-IR in 25 SZ-nonpscyhiatric control (NPC) subject pairs. Points on, above, or below the diagonal line indicate that $\mathrm{SZ}=\mathrm{NPC}, \mathrm{SZ}>\mathrm{NPC}$, and $\mathrm{SZ}<\mathrm{NPC}$ values, respectively. (Inset) Mean MAP2-IR was reduced in deep layer 3 of the primary auditory cortex from SZ subjects. Error bars represent \pm standard error of the mean, and * indicates $p<0.05$

was limited to MAP2-IR(low) subjects, and is due to fewer small spines (Fig. 3d). In contrast, DSD in MAP2-IR(normal) SZ subjects was only modestly, and insignificantly lower (Fig. 3d).

MAP2-IR mediates the effect of SZ on DSD. Given the plausible biological relationship between DSD and MAP2-IR, we hypothesized that MAP2-IR mediates the effect of SZ on DSD. We tested this hypothesis in the combined cohort using the Baron and Kenny framework for mediation, the results are summarized in Table 2. DSD in the smallest three size categories was significantly lower in SZ subjects without controlling for MAP2IR. Similarly, there was a positive effect of MAP2-IR on DSD in the smallest four size categories in the combined cohort when a single meta-analytic average for the effect of MAP2-IR on DSD within each spine size category is used. Finally, the effect of MAP2-IR on DSD was significant in the smallest three size categories after controlling for diagnosis, and the effect of diagnosis on DSD decreased upon the addition of MAP2-IR to the model. Together, these findings support our hypothesis that MAP2-IR mediates the effect of SZ on DSD in the smallest three size categories.

The estimated mediation effect was 0.000902 (52\%), 0.000783 $(46 \%)$, and $0.000448(43 \%)$ in spine size categories 1, 2, and 3, respectively. The $95 \% \mathrm{Cls}$ estimated by bootstrapping were (0.000207, 0.001227), (0.000306, 0.001169), and (0.000099, 0.000687 ) for spine size categories 1,2 , and 3, respectively. The fact that the $95 \%$ bootstrap Cls for the mediation effect did not include zero indicates a statistically significant mediation role for MAP2-IR in the effect of SZ on DSD in the noted categories.

\section{DISCUSSION}

We assessed DSD and MAP2-IR in postmortem deep layer 3 of the primary auditory cortex (A1) in 25 subjects with SZ and 25 NPC subjects, and combined these data with data from two previously studied cohorts for further analysis. We demonstrated that low DSD and low MAP2-IR are robust SZ phenotypes, and showed that neither phenotype is associated with an extensive list of potential confounding variables. We identified a subgroup of SZ subjects with low MAP2-IR and found that lower DSD is limited to these subjects. Finally, we showed that the effect of SZ on DSD is statistically mediated by MAP2-IR.

Our finding that the density of small spines in A1 deep layer 3 is lower in subjects with SZ suggests disruption of the circuits in which pyramidal neurons from this region participate. The spines on these neurons are termination sites for local intracortical efferents and segregate auditory frequency inputs [31]. Fewer small spines on these neurons would presumably result in impaired frequency discrimination. In addition, small spines are enriched for new spines, which serve to remodel cortical circuits so as to instantiate experience-dependent learning $[32,33]$. Thus, our findings suggest a neurobiological mechanism underlying deficits in auditory frequency discrimination and plasticity observed in subjects with SZ [9].

Like low DSD, low MAP2-IR has been reported in several cortical areas in SZ subjects [34-38], leading a recent reviewer to describe low MAP2-IR as a pathologic "hallmark" of SZ [39]. The vast majority of MAP2 is located in the soma and dendrites of neurons. However, MAP2 can be found in dendritic spines and in some non-neuronal cells at low levels [40], and these MAP2 populations may also contribute to the MAP2-IR signal in this study as MAP2-IR was measured in a single tissue plane from multiple sites in deep layer 3 of A1. Thus, neither the types of cells (e.g., pyramidal neurons versus interneurons) nor the cell microdomains (e.g., spines versus dendritic shaft) in which MAP2-IR is lower can be determined from these data. That said, low MAP2-IR in this study likely reflects neuronal somatodendritic MAP2-IR given what is known about the distribution of MAP2 in the human cerebral cortex. Despite the consistent and widespread observation of low MAP2-IR in subjects with SZ, MAP2 protein levels do not differ between SZ and NPC subjects, even in subjects with low MAP2-IR [11], see also Supplemental Fig. 2). Thus, low MAP2-IR in SZ subjects more likely reflects an inability for MAP2 to be detected by antibodies due to changes in the accessibility of the antigenic epitope. Mechanisms by which antigenic epitope accessibility may change include altered interactions of MAP2 protein with other proteins such that the antigenic epitope is obscured and post-translational modification (PTM) of MAP2 protein in a way that induces a conformation change and distorts the antigenic epitope. PTMs are the covalent modification of proteins following protein synthesis. The list of PTMs is extensive and many of the most common, including phosphorylation and glycosylation, have been demonstrated to induce significant change in protein conformation [41].

We found that MAP2-IR statistically mediates the effect of SZ on DSD. DSD was significantly lower in the SZ group, the group effect appears mainly due to low DSD values in a subset of SZ subjects $(60 \%)$ defined as having "low" MAP2-IR based on the distribution of MAP2-IR values in the NPC group. DSD in SZ subjects with "normal" MAP2-IR did not significantly differ from DSD in NPC subjects. Biologically, MAP2 functions as a primary regulator of MT dynamics. MTs undergo changes in length through rapid polymerization and depolymerization [42], and MAP2 binding stabilizes MTs [43, 44]. MAP2 binding affects dendritic outgrowth and morphogenesis as evidenced by the fact that MAP2-deficient mice exhibit reduced MT density in dendrites and reduced dendritic length [45]. MTs invade spines and play a major role in spine development and maintenance [16-18]. MAP2 may influence the invasion of spines by MTs and thus spine dynamics. Indeed, knockdown of the MT plus endbinding protein EB3, which directly binds MAP2 [15] and regulates MT dynamics [46], reduces spine formation and modifies spine morphology [47]. Induction of $\mathrm{N}$-methyl-D-aspartic acid-dependent long-term depression leads to spine shrinkage 
A

NPC

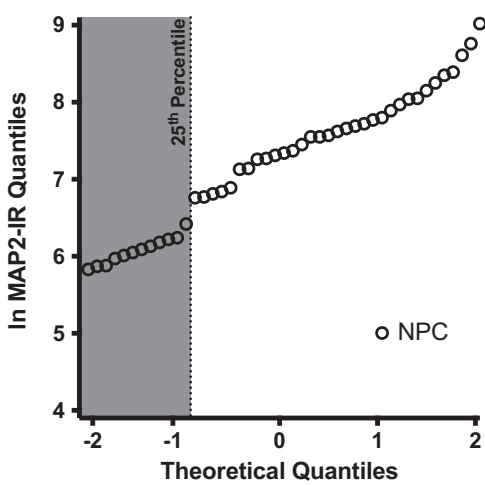

C

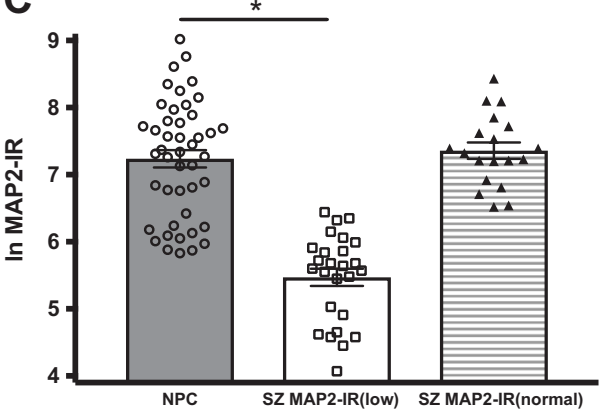

B

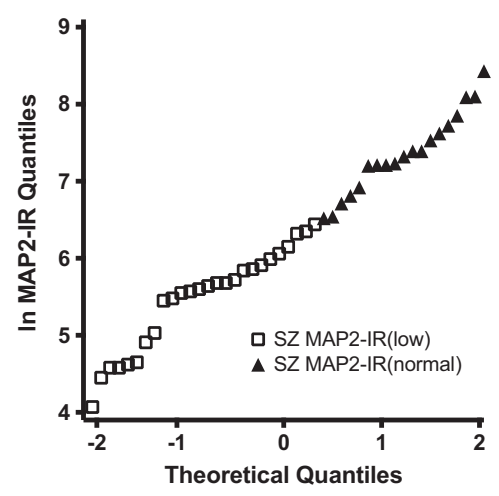

D

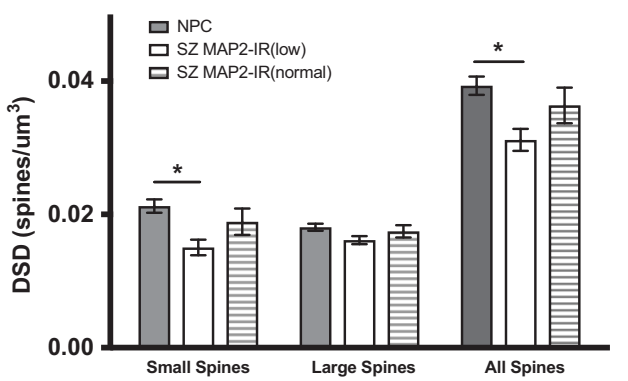

Fig. 3 Lower dendritic spine density in schizophrenia (SZ) subjects is limited to those with low microtubule-associated-protein-2 immunoreactivity, and due to fewer small spines. a, b SZ subjects were divided into two groups based on MAP2-IR values: the SZ MAP-IR(low) group comprised SZ subjects with MAP2-IR values below the 25th percentile for MAP2-IR values in non-psychiatric control (NPC) subjects (In $(M A P 2-I R)=6.52)$, and the SZ MAP-IR(normal) group comprised all the remaining SZ subjects. c Dendritic spine density (DSD) was significantly lower in SZ MAP2-IR(low), but not SZ MAP2-IR(normal), subjects. d Lower DSD in SZ MAP-IR(low) subjects is accounted for by fewer small spines $\left(<0.45 \mu \mathrm{m}^{3}\right)$. Error bars represent \pm standard error of the mean, and ${ }^{*}$ indicates $p<0.05$

via relocalization of EB3 from spines to the dendrite shaft in a MAP2-dependent manner [15]. Combined, these observations suggest that MAP2 dysfunction could be sufficient to alter DSD in neurons. However, the alternative, that impairments leading to lower DSD cause lower MAP2-IR cannot be excluded.

Despite the robustness of our results, the findings from any study of postmortem brain tissue alone are only correlative and cannot establish mechanistic relationships. DSD and MAP2-IR phenotypes in SZ may be due to disease- or treatment-induced changes in the brain or exposure to other confounds. That said, these measures are not affected by antipsychotic exposure in an animal model [10, 11]. Further, our analyses of potential confounding variables in this extensively characterized cohort suggest that many of the potential confounding variables commonly associated with this type of study do not affect DSD or MAP2-IR. In fact, tobacco smoking was the only potentially confounding variable with an effect on DSD that approached statistical significance. For SZ subjects who smoked tobacco, the effect of diagnosis on DSD was blunted in the six smallest spine size categories $\left(<0.90 \mu \mathrm{m}^{3}\right)$ compared nonsmoking SZ subjects $(p \leq 0.1)$, consistent with the theory that individuals with SZ smoke tobacco at high rates because it ameliorates cognitive symptoms [48]. The possible pro-cognitive effects of tobacco smoking are usually attributed to the action of nicotine at neuronal nicotinic receptors [49]; however, recent studies suggest that DNA methylation (DNAm) is another candidate mechanism for this effect. Tobacco smoking leaves a distinct DNAm signature in multiple tissues [50], and recent evidence supports a role for DNAm in the molecular mechanism leading to altered DSD in SZ [51].

\section{CONCLUSION}

We confirmed both low DSD and low MAP2-IR are robust SZ phenotypes, and established MAP2-IR as a statistical mediator of the effect of SZ on DSD. Further, we identified a subgroup of SZ subjects with low MAP2-IR and found that significant alterations in DSD appeared to be more limited to these subjects. Future studies should focus on further characterizing the link between MAP2-IR and DSD (e.g., through phosphorylation of MAP2).

\section{FUNDING AND DISCLOSURE}

This work was supported by NIH Grants K23 MH112798 (BCM), K01 MH107756 (MLM), and R01 MH071533 (RAS). The content is solely the responsibility of the authors and does not necessarily represent the official views of the National Institutes of Health, the Department of Veterans Affairs, or the United States Government. DAL currently receives investigatorinitiated research support from Pfizer, and in 2017 served as a consultant to Merck. All the other authors declare no competing interests.

\section{ADDITIONAL INFORMATION}

Supplementary Information accompanies this paper at (https://doi.org/10.1038/ s41386-019-0350-7).

Publisher's note: Springer Nature remains neutral with regard to jurisdictional claims in published maps and institutional affiliations. 


\section{REFERENCES}

1. Bertling $E$, Hotulainen P. New waves in dendritic spine actin cytoskeleton: from branches and bundles to rings, from actin binding proteins to post-translational modifications. Mol Cell Neurosci. 2017;84:77-84.

2. Bhatt $\mathrm{DH}$, Zhang S, Gan W-B. Dendritic spine dynamics. Annu Rev Physiol. 2009;71:261-82.

3. Holtmaat A, Svoboda K. Experience-dependent structural synaptic plasticity in the mammalian brain. Nat Rev Neurosci. 2009;10:647-58.

4. Chen C-C, Lu J, Zuo Y. Spatiotemporal dynamics of dendritic spines in the living brain. Front Neuroanat. 2014;8:28.

5. Penzes P, Buonanno A, Passafaro M, Sala C, Sweet RA. Developmental vulnerability of synapses and circuits associated with neuropsychiatric disorders. J Neurochem. 2013;126:165-82.

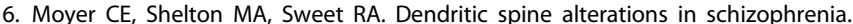
Neurosci Lett. 2015;601:46-53.

7. Glausier JR, Lewis DA. Dendritic spine pathology in schizophrenia. Neuroscience. 2013;251:90-107.

8. Lewis DA, Sweet RA. Schizophrenia from a neural circuitry perspective: advancing toward rational pharmacological therapies. J Clin Invest. 2009;119:706-16.

9. Javitt DC, Sweet RA. Auditory dysfunction in schizophrenia: integrating clinical and basic features. Nat Rev Neurosci. 2015;16:535-50.

10. Sweet RA, Henteleff RA, Zhang W, Sampson AR, Lewis DA. Reduced dendritic spine density in auditory cortex of subjects with schizophrenia. Neuropsychopharmacology. 2009;34:374-89.

11. Shelton MA, Newman JT, Gu H, Sampson AR, Fish KN, MacDonald ML, et al. Loss of microtubule-associated protein 2 immunoreactivity linked to dendritic spine loss in schizophrenia. Biol Psychiatry. 2015;78:374-85.

12. MacDonald ML, Alhassan J, Newman JT, Richard M, Gu H, Kelly RM, et al. Selective loss of smaller spines in schizophrenia. Am J Psychiatry. 2017;174:586-94.

13. Matus A. Microtubule-associated proteins and the determination of neuronal form. J Physiol. 1990;84:134-7.

14. Dehmelt L, Halpain S. The MAP2/Tau family of microtubule-associated proteins. Genome Biol. 2006;7:224.

15. Kapitein LC, Yau KW, Gouveia SM, van der Zwan WA, Wulf PS, Keijzer N, et al NMDA receptor activation suppresses microtubule growth and spine entry. J Neurosci. 2011;31:8194-209.

16. Jaworski J, Kapitein LC, Gouveia SM, Dortland BR, Wulf PS, Grigoriev I, et al. Dynamic microtubules regulate dendritic spine morphology and synaptic plasticity. Neuron. 2009;61:85-100.

17. Gu J, Zheng JQ. Microtubules in dendritic spine development and plasticity Open Neurosci J. 2009;3:128-33.

18. Hu X, Viesselmann C, Nam S, Merriam E, Dent EW. Activity-dependent dynamic microtubule invasion of dendritic spines. J Neurosci. 2008;28:13094-105.

19. Glantz LA, Lewis DA. Decreased dendritic spine density on prefrontal cortical pyramidal neurons in schizophrenia. Arch Gen Psychiatry. 2000;57:65-73.

20. DSM-IV-TR. Diagnostic and statistical manual of mental disorders. DSM-IV-TR; Washington, DC. 2000.

21. Muly EC, Smith Y, Allen P, Greengard P. Subcellular distribution of spinophilin immunolabeling in primate prefrontal cortex: localization to and within dendritic spines. J Comp Neurol. 2004;469:185-97.

22. Allen PB, Ouimet CC, Greengard P. Spinophilin, a novel protein phosphatase 1 binding protein localized to dendritic spines. Proc Natl Acad Sci USA. 1997;94:9956-61.

23. Capani F, Ellisman MH, Martone ME. Filamentous actin is concentrated in specific subpopulations of neuronal and glial structures in rat central nervous system. Brain Res. 2001;923:1-11.

24. Kaufmann WE, Taylor CV, Lishaa NA. Immunoblotting patterns of cytoskeletal dendritic protein expression in human neocortex. Mol Chem Neuropathol. 1997;31:235-44.

25. Kirkwood CM, Ciuchta J, Ikonomovic MD, Fish KN, Abrahamson EE, Murray PS, et al. Dendritic spine density, morphology, and fibrillar actin content surrounding amyloid- $\beta$ plaques in a mouse model of amyloid- $\beta$ deposition. J Neuropathol Exp Neurol. 2013:72:791-800.

26. Rocco BR, Oh H, Shukla R, Mechawar N, Sibille E. Fluorescence-based cell-specific detection for laser-capture microdissection in human brain. Sci Rep. 2017;7:14213.

27. Ridler, T.W. Calvard S. Picture Thresholding using iterative selection methods. IEEE Trans Syst Man Cybern. 1978;8,630-2.

28. Baron RM, Kenny DA. The moderator-mediator variable distinction in social psychological research. conceptual, strategic, and statistical considerations. J Pers Soc Psychol. 1986;51:1173-82.

29. Preacher KJ, Hayes AF. SPSS and SAS procedures for estimating indirect effects in simple mediation models. Behav Res Methods Instrum Comput. 2004;36:717-31.

30. Hayes AF. Beyond Baron and Kenny: statistical mediation analysis in the new millennium. Commun Monogr. 2009.

31. Chen X, Leischner U, Rochefort NL, Nelken I, Konnerth A. Functional mapping of single spines in cortical neurons in vivo. Nature. 2011:475:501-5.

32. Berry KP, Nedivi E. Spine dynamics: are they all the same? Neuron. 2017;96:43-55.

33. Parajuli LK, Tanaka S, Okabe S. Insights into age-old questions of new dendritic spines: From form to function. Brain Res. Bull. 2017;129:3-11.

34. Jones LB, Johnson N, Byne W. Alterations in MAP2 immunocytochemistry in areas 9 and 32 of schizophrenic prefrontal cortex. Psychiatry Res. 2002;114:137-48.

35. Rioux L, Ruscheinsky D, Arnold SE. Microtubule-associated protein MAP2 expression in olfactory bulb in schizophrenia. Psychiatry Res. 2004;128:1-7.

36. Rosoklija G, Keilp JG, Toomayan G, Mancevski B, Haroutunian V, Liu D, et al. Altered subicular MAP2 immunoreactivity in schizophrenia. Prilozi. 2005;26:13-34.

37. Somenarain L, Jones LB. A comparative study of MAP2 immunostaining in areas 9 and 17 in schizophrenia and Huntington chorea. J Psychiatr Res. 2010;44:694-9.

38. Arnold SE, Lee VM, Gur RE, Trojanowski JQ. Abnormal expression of two microtubule-associated proteins (MAP2 and MAP5) in specific subfields of the hippocampal formation in schizophrenia. Proc Natl Acad Sci USA. 1991;88:10850-4

39. Marchisella F, Coffey ET, Hollos P. Microtubule and microtubule associated protein anomalies in psychiatric disease. Cytoskeleton. 2016;73:596-611.

40. Zhang Y, Sloan SA, Clarke LE, Caneda C, Plaza CA, Blumenthal PD, et al. Purification and characterization of progenitor and mature human astrocytes reveals transcriptional and functional differences with mouse. Neuron. 2016;89:37-53.

41. Xin F, Radivojac P. Post-translational modifications induce significant yet not extreme changes to protein structure. Bioinformatics. 2012;28:2905-13.

42. Mitchison T, Kirschner M. Dynamic instability of microtubule growth. Nature. 1984;311:237-42.

43. Pryer NK, Walker Ra, Skeen VP, Bourns BD, Soboeiro MF, Salmon ED. Brain microtubule-associated proteins modulate microtubule dynamic instability in vitro. Real-time observations using video microscopy. J Cell Sci. 1992;103(Part 4):965-76.

44. Takemura R, Okabe S, Umeyama T, Kanai Y, Cowan NJ, Hirokawa N. Increased microtubule stability and alpha tubulin acetylation in cells transfected with microtubule-associated proteins MAP1B, MAP2 or tau. J Cell Sci. 1992;103(Part 4):953-64.

45. Harada A, Teng J, Takei Y, Oguchi K, Hirokawa N. MAP2 is required for dendrite elongation, PKA anchoring in dendrites, and proper PKA signal transduction. J Cell Biol. 2002;158:541-9.

46. Lansbergen G, Akhmanova A. Microtubule plus end: a hub of cellular activities. Traffic. 2006;7:499-507.

47. Gu J, Firestein BL, Zheng JQ. Microtubules in dendritic spine development. J Neurosci. 2008;28:12120-4.

48. Kumari V, Postma P. Nicotine use in schizophrenia: the self medication hypotheses. Neurosci Biobehav Rev. 2005;29:1021-34

49. Winterer G. Why do patients with schizophrenia smoke? Curr Opin Psychiatry. 2010;23:112-9.

50. Joehanes R, Just AC, Marioni RE, Pilling LC, Reynolds LM, Mandaviya PR, et al. Epigenetic signatures of cigarette smoking. Circ Cardiovasc Genet. 2016;9:436-47.

51. McKinney B, Ding $Y$, Lewis DA, Sweet RA. DNA methylation as a putative mechanism for reduced dendritic spine density in the superior temporal gyrus of subjects with schizophrenia. Transl Psychiatry. 2017;7:e1032. 